\title{
Bi-chromatic measurement of thermal fields induced by friction
}

\author{
by E Berté*, J-F. Witz*, X.Boidin*, Y.Desplanques*, and P.Dufrenoy* \\ * Laboratoire de Mécanique de Lille-CNRS UMR 80107, Boulevard Paul Langevin, 59655 Villeneuve d'Ascq \\ cedex, France, jean-francois.witz@ec-lille.fr
}

\begin{abstract}
This study is concerned with a thermal analysis of a braking disc surface for a better understanding of the phenomenas occurring during friction. To discriminate thermal and physico-chemical phenomena, measurements of temperature and emissivity fields are essential during a braking test. This measurement is performed based on a bichromatic thermography technique using two Infrared cameras. To follow the evolution of those phenomenas, the cameras are used to observe the same area of the disc during its deceleration, with a synchronization system . The bichromatic full-field measurements with any technique developed has been produced on a braking test. The results allow a better characterization of the braking.
\end{abstract}

1.

\section{Introduction}

During a pad disc braking the kinetic energy dissipation in heat flux through the contact surfaces is the main cause of degradation of friction elements. The temperature increment leads oxidation, wear, and thermo mechanical deformations which can cause disc cracking [1-3]. In order to improve elements friction behavior with temperature, many temperature measurements techniques are used and developed for braking tests. There are contact measurements technics: using thermocouples to measure mass temperature [4-6], and contact-less optical measurements technics to measure surface temperature: Infrared camera, pyrometer [7-9]. However, infrared temperature measurements involved to know surface emissivity depending on temperature, wave length, roughness, physic-chemical surfaces properties which are changing in braking due to friction and wear mechanisms [10-12]. Simultaneous measurement of temperature and emissivity is possible using poly-chromatic infrared methods. The bi-chromatic pyrometer developed for braking is a useful technics for quantifying temperature, emissivity and braking test analysis [13-14]. The main inconvenient of this method is spot measurement. In order to measure temperature and emissivity field, this method is extended to Focal Plane Array (FPA) detectors. The temperature and emissivity field measurement is performed by the introduction of a bichormatic thermography using two IR cameras with filters. Implementation of this methods involved difficulties on observation of the same disc area by both cameras and measurement of a fast and transient phenomenon [15] as cameras are different in terms of resolution and response time. This study present a strategy and a specific device developed to deal with time synchronization applied to braking tests.

2.

\section{Methodology and experimental means}

Using two IR cameras for bi-chromatic measurements involved difficulties. Firstly, observation of a same area by 2 cameras to get 2 similar images is impossible. The images need to be registered. Secondly, the cameras response times are different also the disc deceleration is non constant. So, to follow thermal phenomena on a disc surface, simultaneous images acquisition must be synchronized with the disc rotation for each cameras.

2.1.

Image registration

As outlined cameras doesn't observe exactly the same disc area. To match both cameras images "Image Registration" technics are used. A displacement field between 2 images one fixed and one moved is calculated using an affine transformation by cross correlation.

In this study the fixed image is an analytic target image chosen to be a random texture at different scales (See Fig. 1) and the moved image correspond to the image of the target obtained by each cameras, respectively. Using an analytic target allows one to correct perspectives. The analytic target made in epoxy resin and copper for a good contrast in the middle wave infrared (See Fig. 2).

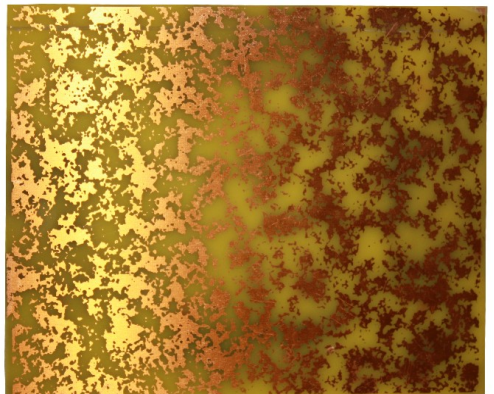

Fig. 1: Picture of the analytical target

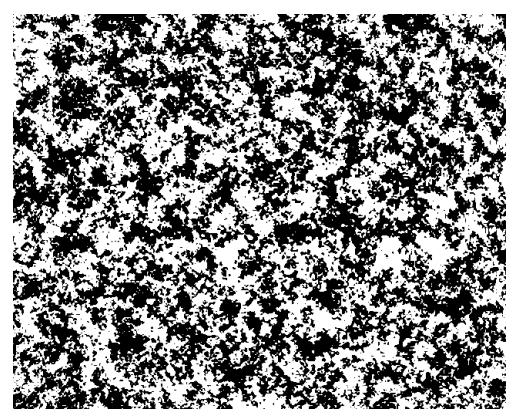

Fig. 2: Analytical target 
Each camera are manually positioned using linear stages in order to get the best observation of the target located in front of the disc before a braking test. Then in post-processing the displacement field is calculated between the original analytic target image and each image of the target given by cameras, with the Image registration method. Thus, each cameras images are match via the analytic image.

To appreciate the image registration the error is calculated by the difference between fixed image and the corrected moved image. If this difference have shade zones the correlation is bad, conversely if there is a good overlay designed the shade zones disappear and the image registration is succeed.

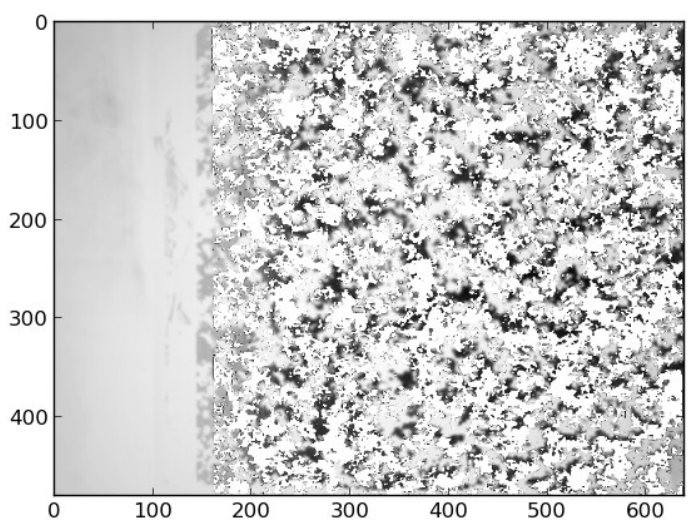

Fig. 3: Error before image registration

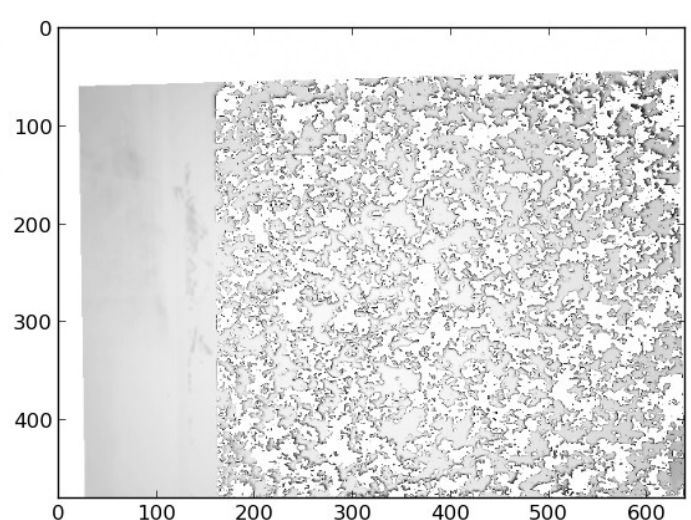

Fig. 4: Error after image registration

2.2.

Temporal synchronization

Cameras have different response time, also phenomena observed are fast with a deceleration depending of the friction factor. To observe thermal flux evolution on the disc surface, it is necessary to synchronize cameras shooting with the disc rotation, in order to have the same angumar position during the braking test.

The solution is to use optical tachymeter to detect $1 / 4$ of disk by the passing of 4 pellets fixed on the flywheel of the tribometer with an $\frac{\Pi}{2}$ angular spacing (see Fig. 5). At each pellet detection the tachymeter send a $10 \mathrm{~V}$ pulse tension to a synchronization unit. The unit synchronization is equipped of a "micro-controller" with an algorithm that determines delays $\left(\Delta t_{d}\right)$ for simultaneous image recording (see Fig. 6) taking into account: speed, deceleration, times responses.

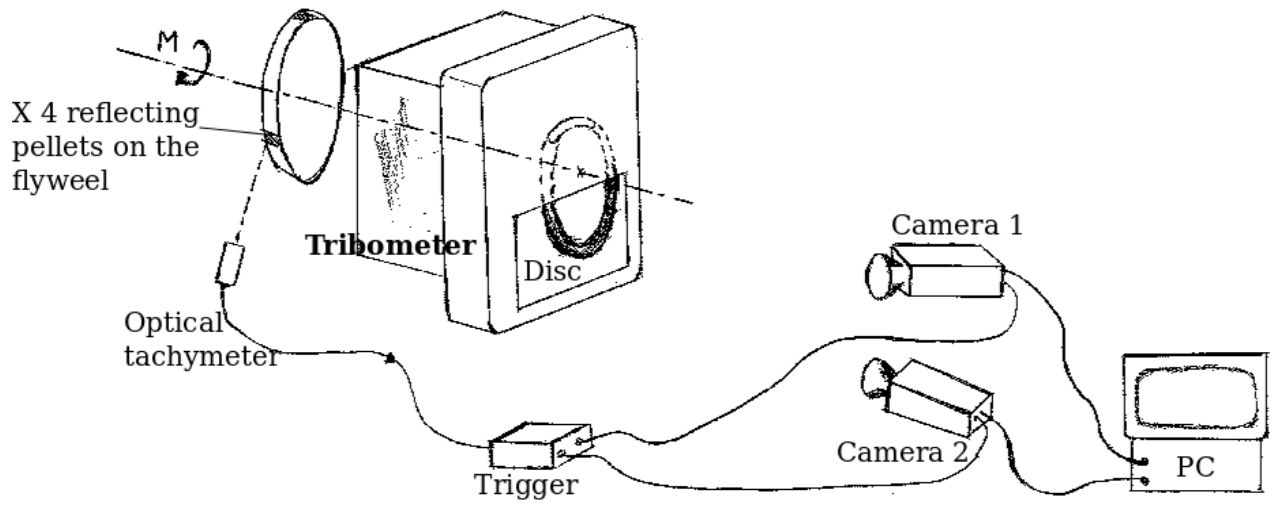

Fig. 5: Synchronisation setup

At the moment when the image should take for a constant $\theta$ the speed of the material point is $\omega_{t}=\omega_{i}+\alpha_{i} \Delta t$ with, $\alpha_{i}=\frac{\omega_{i}-\omega_{i-1}}{t_{i}-t_{i-1}}$, the angular acceleration. 
The mean speed during $\Delta t$ is : $\quad \omega_{\text {moy }}=\frac{\Delta \theta}{\Delta t}=\frac{\omega_{t}+\omega_{i}}{2}=\frac{\omega_{i}+\alpha_{i} \Delta t+\omega_{i}}{2}$

Algorithm looking to solve the motion equation for $\theta$ constant.

$$
\Delta \theta=\omega_{i} \Delta t+\frac{\alpha_{i}}{2} \Delta t^{2}
$$

The solution of equation is :

$$
\Delta t=\frac{-\omega_{i}+\sqrt{\omega_{i}^{2}+2 \alpha_{i} \Delta \theta}}{\alpha_{i}}
$$

corresponding to the time between the detection of a pellet and time to take picture for a $\theta$ constant at each $1 / 4$ turn. Considering a camera, $\Delta t=t_{c a m}+\Delta t_{d}$. With $t_{c a m}$ : the camera response time which is constant. $\Delta t_{d}$ is the delay calculus by algorithm to send trigger signal at camera after the detection of pellet to take the same area at each $1 / 4$ turn.

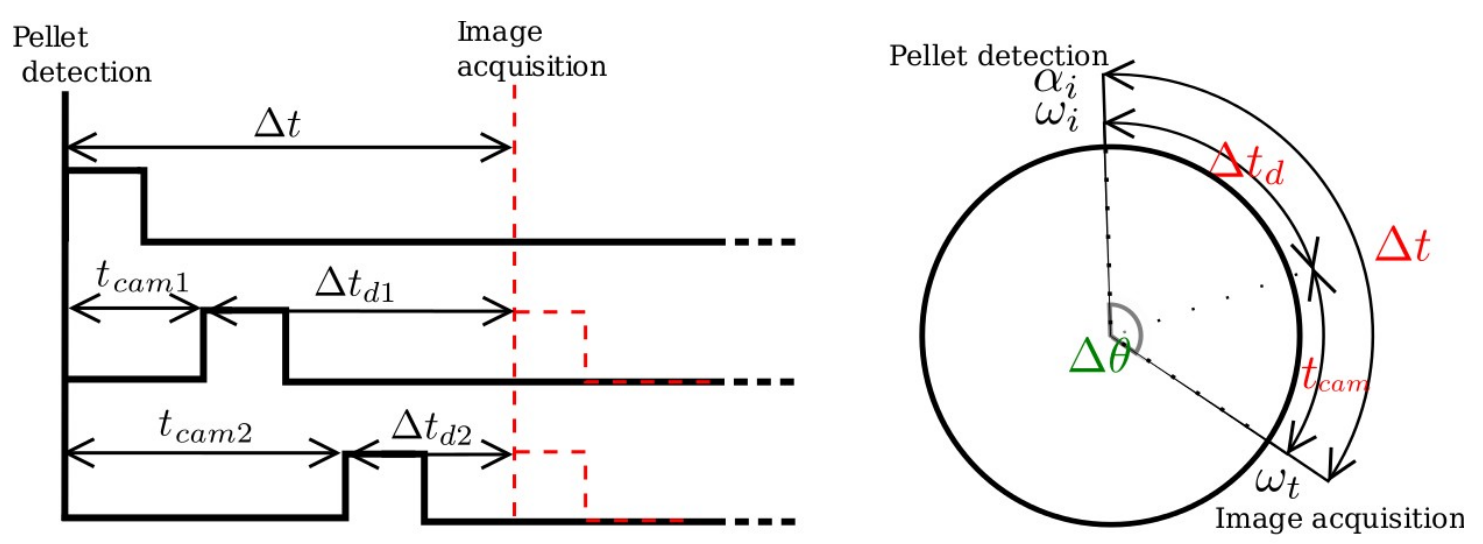

Fig. 6: Synchronization principle

Infrared cameras give an image of the emitted flux in Digital Level (DL) of the observed object. Also each cell of the FPA has a different thermal flux sensibility and camera lens gives rise to a non uniform image. The interest of the calibration is to Link DL of each pixels to a thermal physic variable and correct the non uniformity of the image. Camera calibration protocol, using an extended black body which heat uniformly here in the MWIR spectral range, at different temperature. For each stabilized temperature of the black body a hundred images are saved and averaging to limit noises in order to get the response of the camera.

The bi-chromatic measurement needs a calibration in flow, using the approximation of plank's law the Wien's law since $\lambda . T<<2900 \mu \mathrm{mK}$.

$$
\Phi_{\lambda_{i}}=\epsilon_{\lambda_{i}} \cdot C_{1} \lambda_{i}^{-5} \exp \left(-C_{2} / \lambda_{i} T\right)
$$

With $\mathrm{C}_{1}$ and $\mathrm{C}_{2}$ radiative constants respectively $3,74.10^{-16} \mathrm{~m}^{4} \mathrm{kgs}^{-3}$ and $1,44.10^{-2} \mathrm{Km}$.

To calibrate each pixel the linear response with the black body flux is fit with a 5 order polynomial function. If one uses a lower order polynomial function the error increase on the final results. Under the gray body assumption ( $\epsilon_{\lambda_{1}}=\epsilon_{\lambda_{2}}$ ) it's possible to solve the bi-chromatic equations system for each pixel and get temperature then emissivity :

$$
\left\{\begin{array}{l}
\Phi_{\lambda_{1}}=\epsilon \Phi_{C N \lambda_{1}}=\epsilon \frac{c_{1} \lambda_{1}^{-5}}{\exp \left(\frac{c_{2}}{\lambda_{1} T}\right)} \\
\Phi_{\lambda_{2}}=\epsilon \Phi_{C N, \lambda_{2}}=\epsilon \frac{c_{1} \lambda_{2}^{-5}}{\exp \left(\frac{c_{2}}{\lambda_{2} T}\right)}
\end{array}\right.
$$




$$
\begin{aligned}
& T=\frac{c_{2}\left(\lambda_{1}-\lambda_{2}\right)}{\lambda_{1} \lambda_{2} \ln \frac{\Phi_{C N, \lambda_{1}}}{\Phi_{C N, \lambda_{2}}}\left(\frac{\lambda_{1}}{\lambda_{2}}\right)^{5}} \\
& \epsilon=\frac{\lambda_{i}^{5}}{c_{1}} \Phi_{\lambda_{i}} \exp \frac{c_{2}}{T \lambda_{i}}
\end{aligned}
$$

Relative errors calibration on temperature and emissivity are estimated. For black body temperatures under $200^{\circ} \mathrm{C}$ both relative errors are more than $10 \%$ and over $300^{\circ} \mathrm{C}$ relatives errors are insignificant minus than $1 \%$. So this determines a flow threshold in both wave length for a good measurement of thermal fields.

Braking tests are realized on a pad-disc braking tribometer developed at the "Laboratoire de Mécanique de Lille". This one allows to simulate stop braking at small scale representative of a railway braking [16]. The Fig. 6 show a schema of the tribometer: the disc is driving in rotation by a asynchronous engine at velocities up to $4000 \mathrm{rpm}$ and an hydraulic actuator at the rear of the pad allows to apply a force up to $1200 \mathrm{~N}$. Tribometer instrumentations include a tachymeter to measure speed and a 3D piezoelectric force sensor to measure braking forces that allows the calculus of a friction factor. At this instrumentation 2 IR cameras, an optical tachymeter with the synchronizing unit are added for the bi-chromatic measurement.

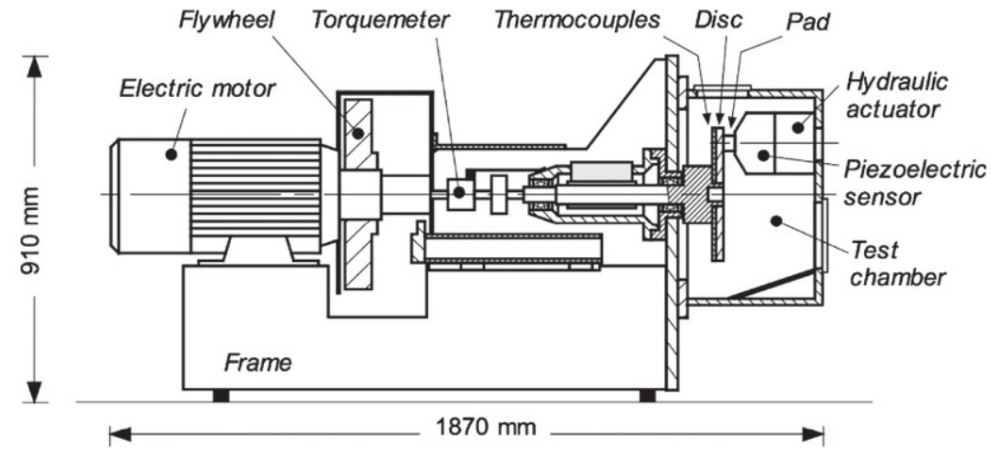

Fig. 7: Schematic drawing of the tribometer

Results presented herein concern the thermal fields of a braking test obtained after post processing with each methods described.

On the Fig. 8 the braking had yet begin since $5 \mathrm{~s}$ and a hot band is migrating to the inner radius. Some circumferential thermal gradient are observable in the same angular locations than bumps of the disc shape at $0^{\circ}, 120^{\circ}$ and $270^{\circ}$ (Fig. 7 ).

This scenario is explained by the fact that during the braking contact locations are on bumps, energy dissipated is important so temperature is very high on these area. Also there are zones where contact seems opened due to disc holes and thermo-mechanical deformations. Thus Wear and oxidation particles could drive softly in opened contact and leads to a high emissivity fields in disc hole.

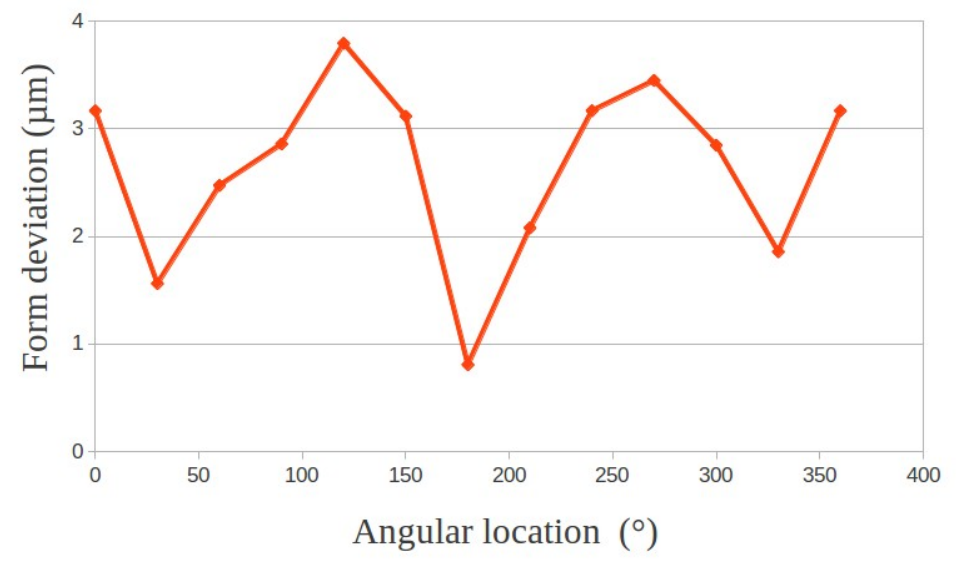

Fig. 7: Initial disc shape 3 bumps undulation, on the tribometer 


\section{Time: $5.055 \mathrm{~s}$}
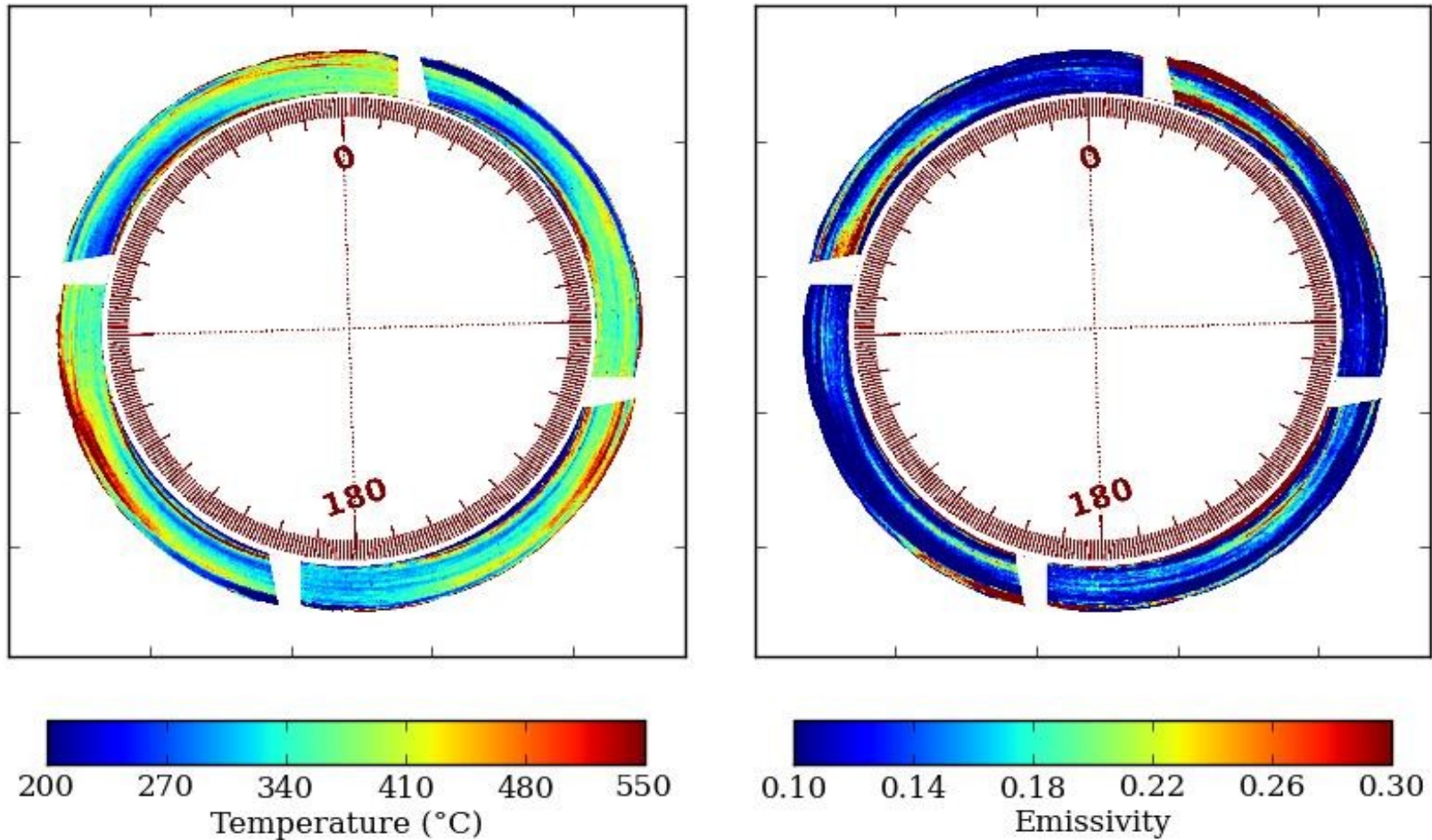

Fig. 8: Temperature and emissivity fields during a braking test

\section{Conclusion}

In this study a measurement tool was developed for thermal field measurements applied to braking tests. The measurement was based on bi-chromatic pyrometry technique, achieved by means of two infrared cameras with filters in different wave lengths. In order too match images of the disc recorded by the both cameras, image registration technics were applied and implemented through an analytical target. Monitoring the evolution of the friction surface of the disc, during a braking test by both cameras, requires time synchronization. The synchronization was made by an algorithm considering acceleration, cameras times responses, for observing 4 same disc sectors at each turn.

The bi-chromatic field measurements with any techniques described has been produced on a braking test, with well-controlled parameters. Assuming the gray body assumption as an admissible one, this method is the first one that allows one to simultaneously observe a temperature field and also an emissivity one. This will lead to new mechanisms analysis in tribology that was not possible before. 


\section{REFERENCES}

[1] E. Francis, Jr. Kennedy Thermal and thermomechanical effects in dry sliding. Wear, 100(1-3):453 -476, 1984.

[2] S. Panier, P. Dufrénoy, and D. Weichert. An experimental investigation of hot spots in railway disc brakes. Wear, 256(7-8):764-773, 2004

[3] P. Dufrénoy, G. Bodovillé, and G. Degallaix. Damage mechanisms and thermomechanical loading of brake discs. In L. Rémy and J. Petit, editors, Temperature-fatigue Interaction International Conference on Temperature-Fatigue Interaction, Ninth International Spring Meeting, volume 29 of European Structural Integrity Society, pages 167-176, 2002.

[4] E. Francis, Jr. Kennedy, D. Frusescu, and J. Li. Thin film thermocouple arrays for sliding surface temperature measurement. Wear, 207(1-2):46-54, 1997.

[5] J. Denape and N. Laraqi Aspect thermique du frottement: mise en évidence expérimentale et élément de modélisation. Mécanique Industries, 1(6):563-579, 2000.

[6] H.S. Qi and A.J. Day. Investigation of disc/pad interface temperatures in friction braking. Wear, 262(5-6):505 513, 2007.

[7] A.-L. Cristol-Bulthé, Y. Desplanques, and G. Degallaix. Coupling between friction physical mechanisms and transient thermal phenomena involved in pad/disc contact during railway braking. Wear, 263(7-12):1230 -1242, 2007.

[8] H. Kasem, S. Bonnamy, Y. Berthier, P. Dufrénoy, and P. Jacquemard. Tribological, physicochemical and thermal study of the abrupt friction transition during carbon/carbon composite friction. Wear, 267(5-8):846- 852, 2009.

[9] M. Siroux, S. Harmand, and B. Desmet. Experimental study using infrared thermography on the convective heat transfer of a tgv brake disk in the actual environment. Optical Engineering 41(7):1558-1564, 2002.

[10] F. Cartigny, R. Copin, M. Siroux, S. Harmand, Y. Desplanques, G. Degallaix., and B. Desmet. Cartographies d'émissivité de la surface d'un disque de frein. Colloque annuel - SFT, pages 351-56, 2000.

[11] B. Desmet, M. Siroux, S. Harmand, F. Cartigny, Y. Desplanques, and G. Degallaix. Métrologie thermique du contact en freinage. Pages 369-376, 2002.

[12] J.Thevenet, M. Siroux, and B. Desmet. Measurements of brake disc surface temperature and emissivity by twocolor pyrometry. Applied Thermal Engineering, 30(6-7):753-759, 2010.

[13] M. Siroux, A-L. Cristol-Bulthé, Y. Desplanques, B. Desmet, and G. Degallaix. Thermal analysis of periodic sliding contact on a braking tribometer. Applied Thermal Engineering, 28(17-18):2194-2202, 2008.

[14] H. Kasem, J. Thevenet, X. Boidin, M. Siroux, P. Dufrenoy, B. Desmet, and Y. Desplanques. An emissivity corrected method for the acurate radiometric measurement of transient surface temperatures during braking. Tribology International, 43(10):1823-1830, 2010.

[15] F. Benilouche, D. Pajani, P. Bremond and P. Potet Thermographie infrarouge d'objet tournant à grande vitesse. Ingénieurs de l'automobile, 667(51-53):51-53, 1992.

[16] R.copin Y. Berthier Y. desplanques, G. Degallaix. A tribometer for the study of materials under railway braking conditions. Tribology Research:From Model Experiment to Industrial Problem, pages 381-391, 2001. 